\title{
Postmarketing nutrivigilance safety profile: a line of dietary food supplements containing red yeast rice for dyslipidemia
}

\author{
Maciej Banach ${ }^{1,2,3}$, Niki Katsiki ${ }^{4}$, Gustavs Latkovskis ${ }^{5,6}$, Manfredi Rizzo ${ }^{7}$, Daniel Pella ${ }^{8}$, \\ Peter E. Penson ${ }^{9,10}$, Zeljko Reiner ${ }^{11}$, Arrigo F.G. Cicero ${ }^{12,13}$
}

\author{
'Polish Mother's Memorial Hospital Research Institute (PMMHRI), Lodz, Poland \\ 2Department of Hypertension, Medical University of Lodz (MUL), Lodz, Poland \\ ${ }^{3}$ Cardiovascular Research Centre, University of Zielona Gora, Zielona Gora, Poland \\ ${ }^{4}$ First Department of Internal Medicine, Diabetes Center, Division of Endocrinology \\ and Metabolism, Medical School, Aristotle University of Thessaloniki, \\ AHEPA Hospital, Thessaloniki, Greece \\ ${ }^{5}$ Institute of Cardiology and Regenerative Medicine, Faculty of Medicine, \\ University of Latvia, Riga, Latvia \\ ${ }^{6}$ Pauls Stradins Clinical University Hospital, Riga, Latvia \\ ${ }^{7}$ Department of Health Promotion Sciences Maternal and Infantile Care, Internal \\ Medicine and Medical Specialties (PROMISE), University of Palermo, Palermo, Italy \\ ${ }^{8} 2^{\text {nd }}$ Department of Cardiology of the East Slovak Institute of Cardiovascular Disease \\ and Faculty of Medicine PJ Safarik University, Kosice, Slovak Republic \\ ${ }^{9}$ School of Pharmacy and Biomolecular Sciences, Liverpool John Moores University, \\ Liverpool, UK \\ ${ }^{10}$ Liverpool Centre for Cardiovascular Science, Liverpool, UK \\ ${ }^{11}$ Department of Internal Medicine, University Hospital Center Zagreb, Zagreb, Croatia \\ ${ }^{12}$ IRCCS Azienda Ospedaliero-Universitaria Di Bologna, Bologna, Italy \\ ${ }^{13}$ Alma Mater Studiorum University of Bologna, Bologna, Italy
}

Submitted: 14 February 2021; Accepted: 26 February 2021

Online publication: 4 March 2021

Arch Med Sci 2021; 17 (4): 856-863

DOI: https://doi.org/10.5114/aoms/133716

Copyright $\odot 2021$ Termedia \& Banach

\section{Abstract}

Introduction: In the absence of a European standardized postmarketing food supplement surveillance system (nutrivigilance), some member states and companies have developed their own approaches to monitoring potential adverse reactions to secure a high level of product safety. This paper describes the use of a nutrivigilance system in monitoring the incidence of spontaneously reported suspected adverse reactions associated with food supplements containing red yeast rice (RYR).

Material and methods: We report the data from a widely used product marketed under the trademark Armolipid/Armolipid Plus. Postmarketing information was collected in a voluntary nutrivigilance system established by the manufacturing company (Meda Pharma SpA, a Viatris Company, Monza, Italy). From $1^{\text {st }}$ October 2004 to $31^{\text {st }}$ December 2019, this system captured cases of suspected adverse reactions spontaneously reported by consumers, healthcare professionals, health authorities, regardless of causality.

Results: The total number of case reports received mentioning the RYR food supplement product line was 542, in which 855 adverse events (AEs) were reported. The total reporting rate of AEs was estimated to be $0.037 \%$ of 2,287,449 exposed consumers. Of the 542 cases, 21 (0.0009\% of exposed consumers) included suspected serious adverse events (SAEs). After careful investigation, 6 cases $(0.0003 \%$ of consumers exposed) and 6 AEs were assessed by the manufacturer as serious and potentially related to exposure to the above-mentioned RYR-based nutraceutical.

\author{
Corresponding author: \\ Prof. Maciej Banach \\ MD, PhD, FNLA, FAHA, \\ FESC, FASA \\ Department \\ of Hypertension \\ Medical University of Lodz \\ 281/289 Rzgowska St \\ 93-338 Lodz, Poland \\ Phone: +48 422711124 \\ E-mail: maciej.banach@ \\ iczmp.edu.pl
}


Conclusions: This nutrivigilance-derived data analysis clearly demonstrates a low prevalence of suspected adverse events associated with the red yeast rice product line. Consumer safety of food supplements could be generally improved by raising awareness of the importance of following the indications and warnings detailed in a food supplement's labeling.

Key words: adverse event, dyslipidaemia, nutrivigilance, red yeast rice, supplement.

\section{Introduction}

Elevated serum low-density lipoprotein cholesterol (LDL-C) is an established risk factor for atherosclerotic cardiovascular (CV) disease [1-3]. For the general population and those at increased CV risk, decreasing the LDL-C by dietary adjustment represents the primary method of CV risk reduction and therefore deserves special emphasis in the evaluation of lifestyle changes [2]. Dietary adjustments, however, achieve a reduction of LDL-C which is insufficient to reach the recommended LDL-C levels in most cases [4].

To this end, the European Society of Cardiology (ESC)/European Atherosclerosis Society (EAS) guidelines 2019 recommend lifestyle interventions, which include not only dietary adjustments but also the use of functional foods [2]. A nutraceutical ingredient, red yeast rice (RYR), has the higher-level (grade A) recommendation because of its clinically relevant impact on improving lipoprotein profiles [2]. The relevance of RYR was discussed in the ESC/EAS guidelines in 2011, together with a recommendation supporting its use. This guidance was reconfirmed in the 2019 update $[2,5]$. Its important role in the lipid-lowering management was also confirmed in the first nutraceuticals guidelines of the International Lipid Expert Panel (ILEP) [6, 7].

For individuals who fall into the low-to-moderate $\mathrm{CV}$ risk category, changes in lifestyle may be indicated, whereas statin therapy may not [8]. The guidelines also state that RYR may be considered for subjects with elevated cholesterol levels for whom statin therapy is not yet indicated [2]. The ESC/EAS guidelines note that clinically relevant cholesterol decreases were reported with RYR amounts containing monacolin $\mathrm{K}$ doses of $2.5 \mathrm{mg}$ / day to $10 \mathrm{mg} /$ day [2].

Several RYR-based supplements are available on the market, some contain only RYR, whereas other products contain additional ingredients [8] such as policosanol [9], berberine [9-11], co-enzyme $\mathrm{Q}_{10}\left(\mathrm{CoQ}_{10}\right)$ [12-14].

In the European Food Safety Authority's (EFSA's) assessment of RYR use (2018), decisions were based on selected studies which evaluated supplementation with mono-ingredient products [15]. The EFSA observed that most reported AEs were musculoskeletal in nature, followed by fatigue, pain, and gastrointestinal (Gl) symptoms [15].
Hepatic AEs were also observed to occur in a significant number of patients receiving RYR supplementation, according to the EFSA [15]. The EFSA considered the lactone form of monacolin $\mathrm{K}$ to be identical to lovastatin and states that RYR food supplement intake could result in an exposure to monacolin $\mathrm{K}$ levels comparable with therapeutic doses of lovastatin [15]. However, taking into account the selectivity of the data sources used in the report, lack of complete data on the quality production, and lack of rechallenge in the included studies, it was difficult to confirm the causality of the reported AEs with the RYR supplementation [16].

A 2017 analysis of 28 brands of RYR supplements available in the United States and the European Union showed that $7 \%$ provided labeling advising against the concurrent use of statins and that monacolin $\mathrm{K}$ content was not included on any of the product labels [17]. In 2 of the analyzed products, monacolin $\mathrm{K}$ was undetectable, and across the brands that did contain monacolin $\mathrm{K}$, the dose per $1200 \mathrm{mg}$ of RYR ranged from $0.09 \mathrm{mg}$ to $5.48 \mathrm{mg}$ [17]. Moreover, the lack of harmonization among nutrivigilance processes and procedures for food supplement products at the EU level further complicates the EU regulatory landscape of food supplements. As a consequence, the reporting requirements of supplement associated AEs, if they exist, may differ from one EU member state to another.

On the contrary, at least 3 controlled trials have shown that RYR is well tolerated [18-20]. In another study, no AEs were reported following 10 weeks, during which 50 patients with dyslipidemia were randomized to a combination food supplement containing monacolin $\mathrm{K}$ (3 mg) or placebo [21]. In a 2012 study, 64 hyperlipidemic patients were randomized to a combination food supplement containing monacolin $\mathrm{K}(3 \mathrm{mg})$ or placebo for 18 weeks, after which no hepatic or renal changes and no serious AEs (SAEs) were reported [22]. In studies that involved a 6- to 48-week course of Armolipid enhanced RYR supplement, $2.2 \%$ of the 1600 treated subjects reported only nonserious AEs, and no life-threatening events were reported. The rates of subjects reporting AEs were not different from placebo [9]. Finally, in a recent meta-analysis that included more than 8500 subjects, RYR supplementation was not associated 
with an increased risk for muscular or non-muscular AEs (which have been observed with statin use); the authors additionally observed significant reduction of SAEs [23].

Based on the abovementioned inconsistent data on RYR safety, the aim of our study was to evaluate the safety of a line of RYR food supplements (Meda Pharma; a Viatris Company, Monza, Italy), available since October 2004, in the postmarketing real-life setting via the company's nutrivigilance and safety data collection methods.

\section{Material and methods}

As an example of a proactive approach, by companies in Europe, to report post-marketing information about safety, we report the data from a widely used product, marketed since $1^{\text {st }}$ October 2004, under the trademark Armolipid and Armolipid Plus, manufactured by Meda Pharma, a Viatris Company (Monza, Italy). Evaluated products were well characterized from a quality perspective (including citrinin content), have customer-friendly labeling, and have comparable formulations (RYR content), allowing the analysis and comparison of postmarketing data while avoiding biases stemming from different contents in the formulations. One tablet of the standard RYR supplement contains RYR (200 mg, the equivalent of monacolin $\mathrm{K}, 3 \mathrm{mg}$ ), folic acid (0.2 mg), $\mathrm{CoQ}_{10}(2 \mathrm{mg})$, and astaxanthin $(0.5 \mathrm{mg})$, and in some countries, policosanol $(10 \mathrm{mg})$. One tablet of the enhanced RYR supplement contains Berberis aristata extract (588 mg, equivalent to berberine chloride $500 \mathrm{mg}$ ), RYR (200 mg, the equivalent of monacolin $\mathrm{K}$, $3 \mathrm{mg})$, policosanol $(10 \mathrm{mg})$, folic acid $(0.2 \mathrm{mg})$, $\mathrm{CoQ}_{10}$ (2.0 mg), and astaxanthin (0.5 mg) [24].

A nutrivigilance process was used to monitor the reporting rate and nature of AEs suspected to be associated with the RYR. An AE was defined as "any untoward medical occurrence" in a consumer while using a food supplement, even when an apparent causal relationship does not necessarily exist. The causal relationship might already have been suspected by the reporter or by the complaining consumer; nevertheless, a causality assessment was performed for all the case reports later in the company's nutrivigilance process. The AEs described on the food supplement label available to the consumer were considered to be "expected" or "labeled". Serious AEs were defined as: death; a life-threatening event; an event that required or prolonged hospitalization; or one that resulted in a significantly or persistently incapacitated or disabled state, a birth defect, or a congenital abnormality. Severe events, based on the definition of the European Medicines Agency (EMA) [25], were not considered synonymous with SAEs.
Since product launch in 2004 an internal nutrivigilance database was developed, with increasing global pharmacovigilance-like systems and procedures. The database was validated and implemented to record case reports from worldwide sources. Spontaneous reports originating from healthcare professionals (HCPs), consumers or health authorities were verified as original source reports (i.e., not duplicates), and AEs were coded according to the standard Medical Dictionary for Regulatory Activities. Collected reports included those, in which a correlation between an AE and a food supplement was suspected by the reporter and was later evaluated for causal association (after entry into the database). Additional collected information included reports of deficient efficacy, misuse (e.g., divergence from label instructions), and contraindicated use during pregnancy or breastfeeding. The World Health Organization-Uppsala Monitoring Centre system was selected to guide the evaluation of causality via parameters including time to onset, clinical plausibility, dechallenge, and rechallenge [24]. Cumulative data reported over a 16-year period (from $1^{\text {st }}$ October 2004 through December 31, 2019) were carefully evaluated and correlated with extrapolated consumer exposure to the RYR food supplement line.

\section{Results}

An estimated total of 2,287,449 consumers from 16 countries (Italy, Spain, Ireland, Germany, Greece, Belgium, Luxemburg, Malta, Hungary, Poland, Russia, Belarus, Ukraine, Thailand, Singapore and Taiwan) took the recommended 1 tablet per day of the Armolipid RYR-based nutraceutical continuously for 1 year. This number was calculated from an estimated annual exposure based on the $834,918,819$ tablets manufactured in the European Union from the time of initial marketing in October 2004 through December 31, 2019, and the recommended dose of 1 tablet per day. The database accumulated 542 spontaneous case reports, estimated to equate to 542 consumers, with 855 suspected AEs. Consumers who reported $\geq 1 \mathrm{AES}$ with respect to use of the RYR were $0.0237 \%$ of the 1-year exposed consumers.

The percentage of any-cause AEs in association with RYR was $0.0374 \%$ of the 1 -year exposed consumers (Table I). The majority of reported AEs were nonserious, with a total of 829 in 855 suspected AEs, which was $97 \%$ of all the AEs and comprised a $0.0362 \%$ prevalence in the exposed consumers. A total of 26 initial SAEs were recorded, received in 21 case reports with $\geq 1$ SAEs. The 21 cases related to 21 different consumers, comprising $0.0009 \%$ of the exposed consumers. Based on the 26 SAEs, a SAE prevalence equal to $0.0011 \%$ of exposed consumers was estimated. 
Table I. Armolipid line red yeast rice-exposed consumers and adverse events

\begin{tabular}{|c|c|c|}
\hline Parameter & Number & $\begin{array}{l}\text { Frequency in exposed } \\
\text { consumers (\%) }\end{array}$ \\
\hline AEs, total & 855 & 0.0374 \\
\hline Consumers reporting $\geq 1 \mathrm{AES}$ & 542 & 0.0237 \\
\hline Nonserious AEs & 829 & 0.0362 \\
\hline AEs reported as serious by reporter & 26 & 0.0011 \\
\hline Consumers reporting an SAE & 21 & 0.0009 \\
\hline $\begin{array}{l}\text { Consumers reporting SAEs confirmed as serious and unable to exclude } \\
\text { causal relationship }\end{array}$ & 6 & 0.0003 \\
\hline Gl AEs, total & 293 & 0.0128 \\
\hline GI SAEs (as reported) & 5 & 0.0002 \\
\hline GI SAEs confirmed as serious and unable to exclude causal relationship & 0 & 0.0000 \\
\hline Musculoskeletal AEs, total & 148 & 0.0064 \\
\hline Musculoskeletal SAEs (as reported) & 2 & 0.0001 \\
\hline $\begin{array}{l}\text { Musculoskeletal SAEs confirmed as serious and unable to exclude causal } \\
\text { relationship }\end{array}$ & 1 & 0.0000 \\
\hline Hepatic AEs (including transaminase alteration), total & 26 & 0.0011 \\
\hline Hepatic SAEs (as reported) & 9 & 0.0004 \\
\hline $\begin{array}{l}\text { Hepatic SAEs confirmed as serious and unable to exclude causal } \\
\text { relationship }\end{array}$ & 3 & 0.0001 \\
\hline Other SOC AEs, total & 388 & 0.0170 \\
\hline Other SOC SAES & 5 & 0.0002 \\
\hline $\begin{array}{l}\text { Other SOC SAEs confirmed as serious and unable to exclude causal } \\
\text { relationship }\end{array}$ & 2 & 0.0001 \\
\hline Consumers exposed & 2287449 & \\
\hline
\end{tabular}

Upon further evaluation, only 6 of the 26 SAEs qualified as serious reactions (fulfilling the established definition) and reactions, in which the food supplement could not be excluded as the cause. This resulted in a SAE frequency of $0.0003 \%$ of exposed consumers.

GI AEs were the most commonly reported (293/855), constituting $34.3 \%$ of the AEs and $0.0128 \%$ of the RYR-based nutraceutical-exposed consumers. Gl-related events included in the 21 case reports of SAEs included reports of diarrhea, vomiting, nausea with vomiting, lipedema, and 1 of intestinal obstruction that was later suspected to be constipation, as it did not require hospitalization and resolved with laxative treatment. The seriousness of the vomiting/nausea reports were assessed as questionable, as was the causality of the lipedema. None of the Gl events reported as serious were confirmed as serious or as resulting only from exposure to this nutraceutical.

Reports of musculoskeletal disorder AEs followed those with Gl features in frequency, comprising 148 of the 855 , or $17.3 \%$ of the AEs, and $0.0064 \%$ of the RYR-exposed consumers. Of the musculoskeletal AEs, 2 were serious in nature but were associated with noncompliance with the recommendations on the supplement's label.
Rhabdomyolysis was reported in 2 consumers (1 involved hospitalization), but in both cases the product's label warnings were not followed. In the first of these 2 cases, an elderly woman who was taking sertraline and rosuvastatin started the enhanced RYR supplement without seeking medical advice. She developed rhabdomyolysis but recovered after discontinuing the RYR supplement and rosuvastatin. The enhanced RYR supplement label advises against its concomitant use with other hypolipidemic products. The second case involved an unknown-gendered consumer who developed rhabdomyolysis and required hospitalization after having started taking the enhanced RYR supplement without prior medical consultation. This subject had a history of rhabdomyolysis in response to simvastatin, and the enhanced RYR supplement label advises consumers to consult a physician to decrease the risk for musculoskeletal AEs. Therefore, no case of rhabdomyolysis without concomitant or prior statin exposure was detected.

Hepatic AEs, including reports of transaminase alterations, occurred in 26 of the 855 reported AEs, constituting $3.0 \%$ of the AEs and a frequency of $0.0011 \%$ in the RYR-exposed consumers. Hepatic SAEs occurred in 7 patients who were diagnosed 
with serious hepatitis. Causality for one of these cases, wherein the subject had an unremarkable medical history and had also been taking atenolol, levothyroxine, and potassium canrenoate, was considered probable by the reporter. In another case, the causality was considered by the reporting physician to be more likely associated with the amoxicillin/clavulanic acid antibiotic (with liver injury as a known adverse drug reaction) also taken by the consumer. In the other SAE reports, various patterns of presentation (i.e., hepatocellular, cholestatic) were mentioned and the symptoms resolved spontaneously or after treatments of glutathione, cortisone, or ursodeoxycholic acid. The latency range in these reports varied widely, from 1 month to 2 years following RYR supplement intake, and the range of causality included probable (in 1 case), possible (in 2 cases), and unlikely (in 2 cases).

The frequency of AEs was calculated, starting from $1^{\text {st }}$ October 2004 through December 31,2017 , then up to December 31, 2019, as was done for the data previously discussed. Up to 2017, consumers of the RYR supplement totalled 1,613,053, and 300 case reports were received mentioning 501 AEs, of which 26 were reported as SAEs by 21 consumers. These data were also shared with the EFSA and with the European Commission Directorate-General for Health and Food Safety as comments to the scientific opinion drafted and published by the EFSA on August 31, 2018. Until the end of 2018, exposed consumers increased to $1,912,475$ and case reports increased to 442, for a total of 705 AEs reported. Still, 26 AEs were reported as serious by 21 consumers. Through the end of 2019, exposed consumers totalled 2,287,449, with 542 case reports mentioning 855 AEs. Again, 26 AEs were reported as serious by 21 consum- ers. Despite the increase in case reports, the number of reports mentioning SAEs has remained unchanged over the past 3 years. Expanding the evaluation up to the past 5 years (Figure 1) showed increases in the number of consumers exposed and in reported AEs. This could be due to increased awareness among HCPs and consumers about the importance of reporting suspected AEs, and possibly as a consequence of the EFSA's published opinion. Nocebo, or more correctly drucebo effect, cannot be also ruled out as a reason of observed AEs [26, 27]. Some factors that also may influence whether an event is reported include length of time since marketing, market share of the suspect product, publicity of the product, and regulatory actions. Also, the mentioned nutrivigilance system showed improved effectiveness over time, with Company's employees trained to systematically forward any case report to the central office of nutrivigilance.

\section{Discussion}

Postmarketing nutrivigilance data showed a high tolerability of the RYR. The percentage of any-cause AE reported when taking RYR was $0.0374 \%$ of the 1 -year-exposed consumers. The majority of reported AEs were nonserious, with a total of 829 of 855 , which was $97 \%$ of all AEs and $0.0362 \%$ of the exposed consumers. Of the 21 case reports (which comprised $0.0009 \%$ of the accumulated exposed consumers) with $\geq 1$ SAEs, 6 qualified as confirmed serious in the nature of the AE and wherein the RYR could not be excluded as the cause, an SAE frequency of $0.0003 \%$ of the exposed consumers. The incidence of AEs reported with the RYR supplement product line were rare to very rare, whereas they are rare to common with lovastatin [8].

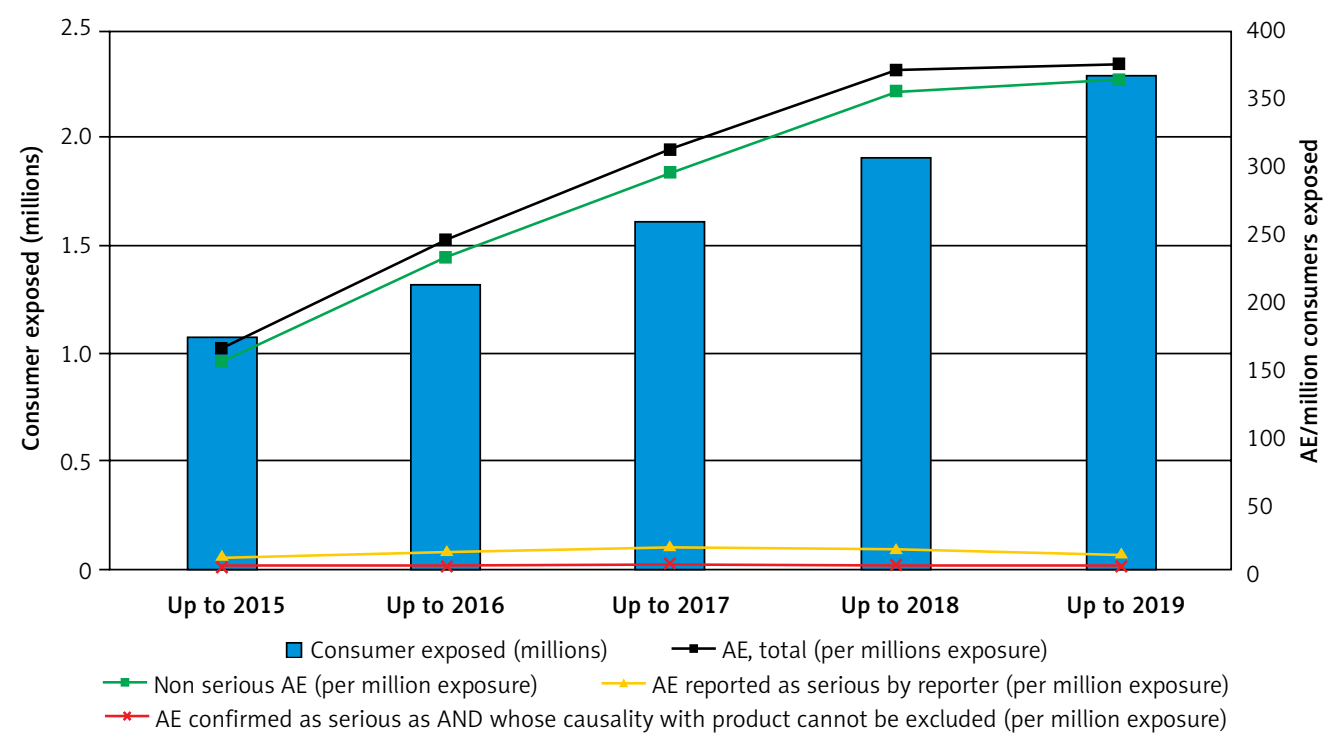

Figure 1. Adverse events on millions of exposed consumers 
The unchanged number of SAEs over the past 3 years demonstrates that the RYR line is well tolerated, with a favorable safety profile when used in line with the product's labeling. The Armolipid RYR line's consumer package leaflets include recommendations regarding dosage, contraindicated conditions (including the concurrent use of drugs for dyslipidemia), and HCP consultation to avoid potential musculoskeletal disorder risk.

Based on the best practices of some European member states (e.g. in Italy, France, and Belgium), the Armolipid RYR has a standardized minimum global label warning that can be further extended based on local authorities' requirements: “Do not exceed the recommended daily dose. Keep out of the reach of young children. Do not use during pregnancy, lactation or in combination with hypolipidemic drugs. It is advised to consult a physician for the product use. Food supplements must not be considered as substitutes of a varied, balanced diet and a healthy lifestyle. Gastrointestinal disorders and muscular pain may occur taking (the Armolipid RYR), mainly in subjects intolerant to statins. A doctor should be consulted if muscular pain occurs".

This evaluation about nutrivigilance had some limitations and strengths. One of the limitations was the inclusion of an RYR product line with a dosage of $3 \mathrm{mg}$ of monacolin $\mathrm{K}$; no other dosages have been monitored, as they do not report regular nutrivigilance data. These methods of reporting cannot be obviously compared with the pharmacovigilance methods, which are implemented for drugs. However, since no vigilance measures/reporting are required from Companies under the current regulations for food supplements/nutraceuticals, we consider this kind of voluntary reporting a good practice, to be widely recommended for monitoring of potential adverse events.

A surveillance assessment reported a collection of suspected AEs from the Italian Surveillance System of Natural Health Products regarding the potential signs of liver injuries and myopathies [16]. This assessment can only be considered qualitatively. In addition, the authors acknowledged the data analyzed had a number of limitations, primarily the unavailability of sales data for RYR food supplements because of their regulatory status (i.e., not reimbursed) [16]. Consequently, such data were not captured in the standard administrative databases to contextualize the number of reported AEs with participant exposure. All supplements that were assessed, except one, contained other natural components than RYR. According to the surveillance authors, the case reports contained limited documentation, lacking information on underlying diseases and suspect- ed concomitant medications. Of importance, such information reported spontaneously from these databases cannot be used to determine incidence rates of AEs [16].

The present results are therefore relevant in demonstrating the importance of a nutrivigilance system applied to food supplements when evaluating the safety of products, once product quality and labeling are established and do not introduce bias. Scientific societies recommend HCPs select products from manufacturers that follow high-level, industry-quality standards [10]. In addition, HCPs are encouraged to report AEs to companies [8]. Companies with standardized products and nutrivigilance systems in place can capture and analyze AE trends for their own products and confirm the products' labeling or evaluate the need for additional warnings to increase consumer awareness.

In conclusion, the implementation of nutrivigilance methods, together with high-quality standards, including control of contaminants, improve consumer safety. Moreover, correct labeling with clearly stated precautions is important for the availability of comprehensive information to consumers. A nutrivigilance system, capturing information spontaneously reported from the markets, also allows manufacturer to confirm the safety of products. Based on the evaluation of the cumulative safety data from the manufacturer's nutrivigilance database, the Armolipid family of RYR supplements has demonstrated that it remains safe, and an effective support for LDL control, as expert guidelines recommend, when it is used according to the instructions on the product label. Compliance with label and/or package leaflet warnings, as well as with healthcare provider (HCP) recommendations, is essential to $A E$ risk reduction, and the overall efficacy and safety of food supplement consumption.

\section{Acknowledgments}

We would like to kindly thank to the Meda Pharma SpA, a Viatris Company (Monza, Italy) for data sharing and providing some help in their analysis and interpretation.

\section{Conflict of interest}

Dr Banach has received research grant(s)/support from Amgen, Mylan, Sanofi and Valeant, and has served as a speaker and consultant for Amgen, Daichii Sankyo, Esperion, Freia Pharmaceuticals, Herbapol, Kogen, KRKA, Mylan, Novartis, Novo Nordisk, Polpharma, Polfarmex, Regeneron, Sanofi-Aventis, Servier, Zentiva; Dr Katsiki has given talks, attended conferences and participated in trials sponsored by Astra Zeneca, Bausch 
Health, Boehringer Ingelheim, Elpen, Menarini, Mylan, Novo Nordisk, Sanofi, Servier and Vianex; Dr. Latkovskis reports grants, honoraria or non-financial support from Abbott Laboratories, Amgen, Astra-Zeneca, Bayer, Berlin-Chemie/Menarini, Boehringer Ingelheim, GlaxoSmithKline, KRKA, Mylan, Novartis, Novo Nordisk, Pfizer, Roche Diagnostics, Sanofi-Aventis, Servier, Siemens Healthcare, Zentiva; Dr Rizzo has given lectures, received honoraria and research support, and participated in conferences, advisory boards and clinical trials sponsored by many pharmaceutical companies including Amgen, AstraZeneca, Boehringer Ingelheim, Kowa, Eli Lilly, Meda, Merck Sharp \& Dohme, Mylan, Novo Nordisk, Novartis, Roche Diagnostics, Sanofi and Servier; he is currently Medical Director, Novo Nordisk BA LM; Dr Penson owns four shares in AstraZeneca PLC and has received honoraria and/or travel reimbursement for events sponsored by AKCEA, Amgen, AMRYT, Link Medical, Napp and Sanofi; Dr Reiner has received honoraria from Sanofi and Novartis. Dr Cicero is a scientific consultant for Meda-Mylan SpA, Sharper SpA and Menarini IFR; Dr Pella has nothing to declare.

\section{References}

1. Grundy SM, Stone NJ, Bailey AL, et al. AHA/ACC/ AACVPR/AAPA/ ABC/ACPM/ADA/AGS/APhA/ASPC/NLA/ PCNA Guideline on the management of blood cholesterol: a Report of the American College of Cardiology/ American Heart Association Task Force on Clinical Practice Guidelines. J Am Coll Cardiol 2019; 73: e285-350.

2. Mach F, Baigent C, Catapano AL, et al. 2019 ESC/EAS Guidelines for the management of dyslipidaemias: lipid modification to reduce cardiovascular risk. Eur Heart J 2020; 41: 111-88.

3. Penson PE, Pirro M, Banach M. LDL-C: lower is better for longer-even at low risk. BMC Med 2020; 18: 320.

4. Ge L, Sadeghirad B, Ball GDC, et al. Comparison of dietary macronutrient patterns of 14 popular named dietary programmes for weight and cardiovascular risk factor reduction in adults: systematic review and network meta-analysis of randomised trials. BMJ 2020; 369: m696.

5. European Association for Cardiovascular Prevention \& Rehabilitation, Reiner Z, Catapano AL, et al. ESC/EAS Guidelines for the management of dyslipidaemias: the Task Force for the management of dyslipidaemias of the European Society of Cardiology (ESC) and the European Atherosclerosis Society (EAS). Eur Heart J 2011; 32: 1769-818.

6. Cicero AFG, Colletti A, Bajraktari G, et al. Lipid lowering nutraceuticals in clinical practice: position paper from an International Lipid Expert Panel. Arch Med Sci 2017; 13: 965-1005.

7. Banach M, Patti AM, Giglio RV, et al. The role of nutraceuticals in statin intolerant patients. J Am Coll Cardiol 2018; 72: 96-118.

8. Banach M, Bruckert E, Descamps OS, et al. The role of red yeast rice (RYR) supplementation in plasma cholesterol control: a review and expert opinion. Atheroscler Suppl 2019; 39: e1-8.
9. Barrios V, Escobar C, Cicero AF, et al. A nutraceutical approach (Armolipid Plus) to reduce total and LDL cholesterol in individuals with mild to moderate dyslipidemia: review of the clinical evidence. Atheroscler Suppl 2017; 24: 1-15.

10. Poli A, Barbagallo CM, Cicero AFG, et al. Nutraceuticals and functional foods for the control of plasma cholesterol levels. An intersociety position paper. Pharmacol Res 2018; 134: 51-60.

11. Pirro M, Mannarino MR, Bianconi $V$, et al. The effects of a nutraceutical combination on plasma lipids and glucose: a systematic review and meta-analysis of randomized controlled trials. Pharmacol Res 2016; 110: 76-88.

12. Mazza A, Lenti S, Schiavon L, et al. Effect of Monacolin $\mathrm{K}$ and $\mathrm{COQ} 10$ supplementation in hypertensive and hypercholesterolemic subjects with metabolic syndrome. Biomed Pharmacother 2018; 105: 992-6.

13. Mazidi M, Kengne AP, Banach M; Lipid and Blood Pressure Meta-analysis Collaboration Group. Effects of coenzyme Q10 supplementation on plasma C-reactive protein concentrations: a systematic review and metaanalysis of randomized controlled trials. Pharmacol Res 2018; 128: 130-6.

14. Banach M, Serban C, Sahebkar A, et al. Effects of coenzyme Q10 on statin-induced myopathy: a meta-analysis of randomized controlled trials. Mayo Clin Proc 2015; 90: 24-34

15. EFSA Panel on Food Additives and Nutrient Sources added to Food (ANS), Younes M, Aggett P, et al. Scientific opinion on the safety of monacolins in red yeast rice. EFSA J 2018; 16: e05368.

16. Mazzanti G, Moro PA, Raschi E. Adverse reactions to dietary supplements containing red yeast rice: assessment of cases from the Italian surveillance system. Br J Clin Pharmacol 2017; 83: 894-908.

17. Cohen PA, Avula B, Khan IA. Variability in strength of red yeast rice supplements purchased from mainstream retailers. Eur J Prev Cardiol 2017; 24: 1431-4.

18. Lu Z, Kou W, Du B, et al. Effect of Xuezhikang, an extract from red yeast Chinese rice, on coronary events in a Chinese population with previous myocardial infarction. Am J Cardiol 2008; 101: 1689-93.

19. Gheith O, Sheashaa H, Abdelsalam M. Efficacy and safety of Monascus purpureus Went rice in subjects with secondary hyperlipidemia. Clin Exp Nephrol 2008; 12: 189-94.

20. Lin CC, Li TC, Lai MM. Efficacy and safety of Monascus purpureus Went rice in subjects with hyperlipidemia. Eur J Endocrinol 2005; 153: 679-86.

21. Affuso F, Ruvolo A, Micillo F. Effects of a nutraceutical combination (berberine, red yeast rice and policosanols) on lipid levels and endothelial function randomized, double-blind, placebo-controlled study. Nutr Metab Cardiovasc Dis 2010; 20: 656-61.

22. Affuso F, Mercurio V, Ruvolo A, et al. A nutraceutical combination improves insulin sensitivity in patients with metabolic syndrome. World I Cardiol 2012; 4: 77-83.

23. Fogacci F, Banach M, Mikhailidis DP, et al. Safety of red yeast rice supplementation: a systematic review and meta-analysis of randomized controlled trials. Pharmacol Res 2019; 143: 1-16.

24. The use of the WHO-UMC system for standardised case causality assessment. Uppsala Monitoring Centre. www.who-umc.org/media/164200/who-umc-causality-assessment_new-logo.pdf. Published 2018. Accessed May 29, 2020. 
25. Clinical Safety Data Management: Definitions and Standards for Expedited Reporting. https://www.ema. europa.eu/en/documents/scientific-guideline/international-conference-harmonisation-technical-requirements-registration-pharmaceuticals-human-use_en15.pdf. Published June 1995. Accessed June 3, 2020.

26. Penson PE, Mancini GBJ, Toth PP, et al. Introducing the 'Drucebo' effect in statin therapy: a systematic review of studies comparing reported rates of statin-associated muscle symptoms, under blinded and open-label conditions. J Cachexia Sarcopenia Muscle 2018; 9: 1023-33.

27. Banach M, Penson PE. Drucebo effect - the challenge we should all definitely face! Arch Med Sci 2021; 17: 542-3. 